\title{
Características do ejaculado de caprinos sob estresse calórico em câmara bioclimática
}

[Sperm characteristics of bucks under heat stress in climatic chamber]

\author{
L.A. Coelho ${ }^{1}$, A. Sasa ${ }^{1}$, C.E. Nader ${ }^{1}$, E.C.C. Celeguini ${ }^{2}$ \\ ${ }^{1}$ Faculdade de Zootecnia e Engenharia de Alimentos - USP \\ Av. Duque de Caxias Norte, 225 \\ 13630-000 - Pirassununga, SP \\ ${ }^{2}$ Faculdade de Medicina Veterinária e Zootecnia - USP - São Paulo, SP
}

\begin{abstract}
RESUMO
Para verificar o efeito do estresse calórico (EC) na produção espermática de caprinos, oito machos das raças Saanen $(n=4)$ e Pardo-Alpina $(n=4)$ foram mantidos em câmara bioclimática, sob condições de termoneutralidade $\left(13,0^{\circ} \mathrm{C}\right.$ a $\left.26,7^{\circ} \mathrm{C}\right)$ durante 30 dias e, após um período (60 dias) de descanso, submetidos ao EC $\left(23,7^{\circ} \mathrm{C}\right.$ a $\left.34,0^{\circ} \mathrm{C}\right)$ por mais 30 dias. Para minimizar as variações sazonais na produção espermática, durante todo o período, o fotoperíodo foi controlado utilizando-se alternância de dias longos (16 horas de luz e 8 horas de escuro) e de dias curtos ( 8 horas de luz e 16 horas de escuro) a cada 30 dias. Avaliaram-se as temperaturas retal e testicular, o volume do ejaculado, a concentração espermática, as motilidades massal e individual progressiva (MIP), o vigor e a morfologia espermática. Houve aumento $(\mathrm{P}<0,05)$ da temperatura do testículo $\left(31,0 \pm 1,1\right.$ vs. $\left.32,8 \pm 0,9^{\circ} \mathrm{C}\right)$ e decréscimos $(\mathrm{P}<0,01)$ do volume $(0,6 \pm 0,3$ vs. $0,4 \pm 0,3 \mathrm{ml})$, da concentração espermática $\left(5,1 \pm 1,8\right.$ vs. $\left.4,5 \pm 1,5 \times 10^{9}\right)$, da motilidade massal $(3,5 \pm 0,5$ vs. $2,9 \pm 0,5)$, da MIP $(67,4 \pm 14,3$ vs. $53,3 \pm 13,1 \%)$ e do vigor $(3,5 \pm 0,6$ vs.3,0 $\pm 0,6)$ quando os animais foram submetidos ao EC. O EC não influenciou $(\mathrm{P}>0,05)$ o percentual total de células anormais e nem a temperatura retal. Os machos da raça Saanen apresentaram temperaturas do testículo e retal mais elevadas $(\mathrm{P}<0,01)$ e produziram maior volume $(\mathrm{P}<0,05)$ de ejaculado. $\mathrm{O}$ estresse calórico produzido em câmara bioclimática foi suficiente para afetar, negativamente, algumas características quanti-qualitativas do ejaculado de machos caprinos das raças Saanen e Pardo-Alpina.
\end{abstract}

Palavras-chave: caprino, sêmen, temperatura ambiente, estresse térmico

\begin{abstract}
To verify the effect of heat stress (HS) on caprine semen production eight male goats of Saanen $(n=4)$ and Alpine Brown $(n=4)$ breeds were kept in climate chamber under thermal neutral conditions $\left(13.0^{\circ} \mathrm{C}\right.$ to $\left.26.7^{\circ} \mathrm{C}\right)$ for 30 days. After a resting period (60 days), the same bucks were submitted to heat stress $\left(23.7^{\circ} \mathrm{C}\right.$ to $\left.34.0^{\circ} \mathrm{C}\right)$ for another 30 days. To neutralize the seasonal variations of sperm production throughout the period, the photoperiod was controlled every 30 days alterning long days (16 hours of light and 8 hours of darkness) and short days (8 hours of light and 16 hours of darkness). The following variables were evaluated: rectal and testicular temperatures, volume of ejaculate, sperm concentration, mass motility, individual progressive motility, vigor and sperm morphology. There was an elevation $(P<0.01)$ of testicular temperature $\left(31.0 \pm 1.1\right.$ vs. $\left.32.8 \pm 0.9^{\circ} \mathrm{C}\right)$ and a decline of volume $(0.6 \pm 0.3$ vs. $0.4 \pm 0.3 \mathrm{ml}$ ), sperm concentration ( $\left.5.1 \pm 1.8 \mathrm{vs} .4 .5 \pm 1.5 \times 10^{9}\right)$, mass motility ( $\left.3.5 \pm 0.5 \mathrm{vs} .2 .9 \pm 0.5\right)$, individual

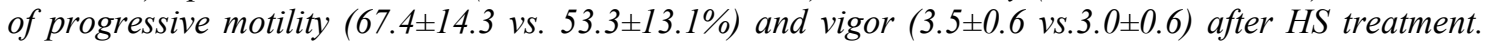
There was no effect of $H S(P>0.05)$ on percentage of morphologically normal spermatozoa and rectal temperature. The Saanen males showed higher testicular and rectal temperatures and produced more
\end{abstract}

Recebido em 6 de dezembro de 2004

Aceito em 2 de maio de 2006

E-mail: liac@usp.com.br

Apoio: FAPESP 
sperm volume than Alpine Brown males. The HS in climate chamber was sufficient to negatively affect some semen characteristics of Saanen and Alpine Brown male goats.

Keywords: caprine, semen, environmental temperature, heat stress

\section{INTRODUÇ̃̃O}

A produção espermática dos caprinos é influenciada por fatores como raça, idade, nutrição, fotoperíodo, temperatura ambiente e umidade, que são responsáveis pela variação das características do sêmen (Chemineau, 2004).

Variações estacionais na quantidade e qualidade do sêmen têm sido atribuídas ao fotoperíodo (Pelletier et al., 1988), com diferenças entre raças em função da latitude do local de medição. Em altas latitudes, a atividade reprodutiva dos machos pode estar ausente por alguns meses (Corteel, 1975; Saumander e Rouger, 1984; Ahmad e Noakes, 1996), enquanto em latitudes intermediárias as variações seminais são menos pronunciadas (Roca et al., 1992; Pérez e Mateos, 1996; Karagiannidis et al., 2000). Em regiões tropicais, próximas à linha do Equador, onde não há variação da luminosidade diária, não ocorrem diferenças estacionais na produção espermática dos machos de raças nativas (Chemineau, 1986; Hibbert el at., 1986). Nessas áreas, as variações quanti-qualitativas do ejaculado caprino parecem estar condicionadas a outros fatores mais importantes do que o fotoperíodo, tais como a temperatura ambiente (Nunes et al., 1988; Machado et al., 2000). Pesquisas têm demonstrado que elevadas temperaturas ambientais podem interferir negativamente na qualidade espermática de ruminantes, sendo a motilidade individual progressiva e o percentual de células morfologicamente anormais as características seminais mais afetadas (Kraemer, 2000; Chemineau, 2004; Valle et al., 2005).

Na Região Sudeste do Brasil, é possível observar variação estacional da atividade reprodutiva dos machos das raças caprinas mais difundidas, a Saanen e a Pardo-Alpina (Espeschit, 1998), mas não existe nenhum estudo sistemático sobre o efeito isolado da temperatura sobre as características do ejaculado. Este trabalho teve como objetivo verificar possíveis variações das características quanti-qualitativas do sêmen de caprinos das raças Saanen e Pardo-Alpina, criados no estado de São Paulo, quando submetidos ao estresse calórico em câmara bioclimática.

\section{MATERIAL E MÉTODOS}

O trabalho foi realizado na Câmara Bioclimática do Laboratório de Biometeorologia e Etologia da USP, Campus de Pirassununga-SP, entre janeiro e agosto de 2002. Foram utilizados oito machos, sendo quatro da raça Pardo-Alpina e quatro da raça Saanen, entre 12 e 18 meses de idade, mantidos em gaiolas metabólicas individuais com $2 \mathrm{~m}^{2} /$ animal, piso de estrado plástico e acesso ao cocho e bebedouro. A dieta fornecida consistia de feno de capim-coast-cross (Cynodon dactylon), duas vezes ao dia, sal mineral para caprinos à vontade e concentrado, 250 $\mathrm{g} /$ animal/dia, com $22 \%$ de proteína bruta, uma vez ao dia, conforme os requisitos nutricionais para ovinos em reprodução (Agricultural..., 1998).

O procedimento experimental teve um período de adaptação de 30 dias, em condições de termoneutralidade, para os animais se ajustarem às condições físicas e de alimentação nas gaiolas dentro da câmara bioclimática. A seguir, os dados foram obtidos durante 30 dias, em condições de termoneutralidade. Após esse período, promoveu-se um descanso de 60 dias correspondendo a, pelo menos, um ciclo espermatogênico, após o qual os dados foram obtidos por mais 30 dias em condições de estresse calórico. Os animais foram submetidos ao estresse das 8 às 17 horas.

Durante o período de adaptação e em todo o período experimental, os valores médios, mínimos e máximos, referentes à temperatura ambiente e à umidade do ar, foram registrados por meio de termoigrógrafos, higrômetros e termômetros de leitura instantânea. As médias mínimas e máximas referentes à temperatura radiante também foram mensuradas por meio de termômetros de globo negro (Tab. 1). 
Tabela 1. Médias \pm desvios-padrão do ambiente térmico durante o período experimental

\begin{tabular}{lcccc}
\hline \multirow{2}{*}{ Variável } & \multicolumn{2}{c}{ Termoneutralidade } & \multicolumn{2}{c}{ Estresse calórico } \\
\cline { 2 - 5 } & Mínima & Máxima & Mínimo & Máximo \\
\hline Temperatura do ar, ${ }^{\circ} \mathrm{C}$ & $13,0 \pm 2,6$ & $26,7 \pm 2,3$ & $23,7 \pm 1,3$ & $34,0 \pm 2,3$ \\
Umidade relativa, $\%$ & $33,6 \pm 11,7$ & $88,8 \pm 6,9$ & $36,6 \pm 12,2$ & $77,9 \pm 6,2$ \\
Temperatura do globo negro, ${ }^{\circ} \mathrm{C}$ & $20,2 \pm 4,5$ & $25,3 \pm 3,0$ & $30,2 \pm 2,5$ & $33,5 \pm 3,1$ \\
\hline
\end{tabular}

Com a finalidade de minimizar as variações sazonais na produção espermática, o fotoperíodo foi controlado através de timer com fornecimento de luz em esquema de alternância de dias longos, 16 horas de luz artificial e 8 horas de escuro, e de dias curtos, 8 horas de escuro e 16 horas de luz artificial, a cada 30 dias, conforme preconizado por Delgadillo et al. (1992).

Após o período de adaptação, os dados foram obtidos duas vezes por semana, sempre pela manhã, sendo avaliadas as temperaturas retal e testicular e dados referentes às seguintes características quanti-qualitativa do ejaculado: volume $(\mathrm{ml})$, concentração espermática x $10^{4}$ motilidade massal (1 a 5), motilidade individual progressiva (MIP), (0 a 100\%), vigor (1 a 5) e morfologia espermática, representada pelo porcentual total de células anormais.

As temperaturas retal e testicular foram medidas por meio de termômetros clínico e digital, respectivamente. $\mathrm{O}$ ejaculado foi colhido por vagina artificial, tendo como manequim uma fêmea estrogenada. Após a colheita do ejaculado, o volume foi determinado em tubo de vidro graduado e o sêmen mantido em banho-maria a $37^{\circ} \mathrm{C}$, para avaliação das demais variáveis. Para a concentração espermática, uma amostra de sêmen diluída em solução formol-salina, na proporção de 1:200, foi avaliada em câmara de Neubauer e expressa em número de espermatozóides x $10^{9}$. Para observação da motilidade massal, uma gota de sêmen não diluído foi colocada em lâmina de vidro e examinada sob microscopia ótica convencional, atribuindo-se valores de 1 a 5 . O ejaculado foi diluído em leite em pó desnatado e glicosado. As avaliações da motilidade individual progressiva e de vigor foram realizadas mediante a observação de uma gota de sêmen diluído, sob lâmina e lamínula, e classificadas em porcentagem e numa escala de 1 a 5, respectivamente. A avaliação da morfologia espermática foi realizada em preparações úmidas não coradas de sêmen fresco fixado em solução formol-salina. Cerca de 200 células de cada preparação foram contadas sob microscopia de contraste de fase, utilizando-se um aumento de 1000X. Anormalidades morfológicas foram grupadas em porcentuais de defeitos maiores e menores, perfazendo o total de defeitos, conforme Blom (1973).

Os dados foram avaliados mediante análise de variância, utilizando-se o procedimento ONEWAY ANOVA (User's..., 1996), seguido pelo teste Duncan para estabelecer a comparação entre médias.

\section{RESULTADOS E DISCUSSÃO}

As temperaturas retal e testicular são mostradas na Tab. 2. A temperatura retal variou com a raça $(\mathrm{P}<0,01)$, mas não foi influenciada $(\mathrm{P}>0,05)$ pelo estresse calórico. Na raça Saanen, as médias de temperatura retal foram mais elevadas que na raça Pardo-Alpina, sugerindo que o estresse calórico não foi suficiente para alterar a temperatura retal segundo a raça, embora os animais Saanen tenham sido mais susceptíveis às modificações das condições ambientais do que os Pardo-Alpina (Tab. 2). A temperatura retal tem sido largamente utilizada como parâmetro para medir o grau de tolerância dos indivíduos ao estresse calórico (Uribe-Velásquez et al., 1998; Chemineau, 2004). Segundo Turner (1982) e Arruda e Pant (1984), as médias das temperaturas retais mantêm-se dentro dos valores considerados fisiológicos para a espécie em condições de termoneutralidade (Arruda e Pant, 1984; Uribe-Velásquez et al., 1998).

$\mathrm{O}$ estresse calórico influenciou $(\mathrm{P}<0,01)$ a temperatura testicular, provocando elevação média de $1,5^{\circ} \mathrm{C}$ ao final do tratamento (Tab. 2). Essa característica também variou $(\mathrm{P}<0,01)$ em função da raça, sendo os valores mais elevados na Saanen. Esses resultados demonstram que os machos Saanen são menos tolerantes à elevação da temperatura ambiente. $\mathrm{O}$ aumento da temperatura testicular como fator responsável 
por alterações de algumas características do ejaculado é um fato bem definido (Kastelic et al., 2000; Kraemer, 2000). Pesquisas com a técnica de insulação do escroto em ruminantes, como modelo para o aumento da temperatura testicular, evidenciaram que as alterações seminais, após a adoção desse procedimento, caracterizaram-se por decréscimos na concentração espermática, na motilidade individual progressiva e no número de células espermáticas normais (Santos e Simplício, 2000; Moreira et al., 2001).

O estresse calórico promoveu alterações em quase todas as características seminais avaliadas, exceto no percentual total de células anormais (Tab. 3). Houve diminuição $(\mathrm{P}<0,01)$ do volume e da concentração espermática, da motilidade massal, da motilidade individual progressiva e do vigor. Estes resultados são semelhantes aos descritos por Kishore e Rao (1983) em caprinos nativos e da raça Saanen, na Índia, por Santos e Simplício (2000), em caprinos mestiços Pardo-
Alpino-Moxotó e da raça Moxotó, e por Morena et al. (2001), em ovinos deslanados no Nordeste do Brasil.

Tabela 2. Médias \pm desvios-padrão das temperaturas retal e testicular, de machos caprinos das raças Pardo-Alpina e Saanen, submetidos a condições de termoneutralidade (TN) e posteriormente ao estresse calórico (EC) em câmara bioclimática

\begin{tabular}{lcc}
\hline \multirow{2}{*}{ Variável } & \multicolumn{2}{c}{ Temperaturas, ${ }^{\circ} \mathrm{C}$} \\
\cline { 2 - 3 } & Retal & Testicular \\
\hline TN & $38,88 \pm 0,4$ & $31,03 \pm 1,1 \mathrm{~b}$ \\
EC & $38,82 \pm 0,3$ & $32,52 \pm 0,6 \mathrm{a}$ \\
Média & $38,85 \pm 0,4$ & $31,78 \pm 0,9$ \\
Raça & & \\
Pardo-Alpina & $38,77 \pm 0,4 \mathrm{~b}$ & $31,56 \pm 1,2 \mathrm{~b}$ \\
Saanen & $38,93 \pm 0,3 \mathrm{a}$ & $31,99 \pm 1,0 \mathrm{a}$ \\
Média & $38,85 \pm 0,4$ & $31,78 \pm 1,1$ \\
\hline
\end{tabular}

Médias seguidas de letras distintas na coluna diferem entre si pelo teste Duncan $(\mathrm{P}<0,05$ e $\mathrm{P}<0,001)$.

Tabela 3. Médias \pm desvios-padrão das características quanti-qualitativas do sêmen de machos caprinos das raças Pardo-Alpina e Saanen, submetidos a condições de termoneutralidade (TN) e posteriormente ao estresse calórico (EC) em câmara bioclimática

\begin{tabular}{lcccccc}
\hline Variável & $\begin{array}{c}\text { VL } \\
(\mathrm{mL})\end{array}$ & $\begin{array}{c}\mathrm{CE} \\
(\mathrm{x} \mathrm{10})\end{array}$ & $\begin{array}{c}\text { MM } \\
(0-5)\end{array}$ & $\begin{array}{c}\text { MIP } \\
(0-100 \%)\end{array}$ & $\begin{array}{c}\text { VG } \\
(0-5)\end{array}$ & $\begin{array}{c}\text { TCA } \\
(\%)\end{array}$ \\
\hline TN & $0,6 \pm 0,3 \mathrm{a}$ & $5,1 \pm 1,8 \mathrm{a}$ & $3,5 \pm 0,5 \mathrm{a}$ & $67,4 \pm 14,3 \mathrm{a}$ & $3,5 \pm 0,6 \mathrm{a}$ & $16,6 \pm 12,8$ \\
ET & $0,4 \pm 0,3 \mathrm{~b}$ & $4,5 \pm 1,5 \mathrm{~b}$ & $2,9 \pm 0,5 \mathrm{~b}$ & $53,3 \pm 13,1 \mathrm{~b}$ & $3,0 \pm 0,6 \mathrm{~b}$ & $18,6 \pm 15,5$ \\
Raça & & & & & & \\
Alpina & $0,5 \pm 0,3 \mathrm{a}$ & $504,1 \pm 193,8$ & $3,2 \pm 0,5$ & $67,5 \pm 15,3$ & $3,3 \pm 0,6$ & $17,3 \pm 15,5$ \\
Saanen & $0,6 \pm 0,3 \mathrm{~b}$ & $463,6 \pm 140,5$ & $3,1 \pm 0,7$ & $54,6 \pm 15,6$ & $3,3 \pm 0,7$ & $17,9 \pm 12,9$ \\
\hline
\end{tabular}

Médias seguidas de letras distintas na coluna diferem entre si pelo teste Duncan $(\mathrm{P}<0,05$ e $\mathrm{P}<0,001)$.

$\mathrm{VL}=$ volume; $\mathrm{CE}=$ concentração espermática; $\mathrm{MM}=$ motilidade massal; $\mathrm{MIP}=$ motilidade individual progressiva; $\mathrm{VG}=$ vigor; $\mathrm{TCA}=$ total de células anormais.

Diferenças entre raças $(\mathrm{P}<0,01)$ foram observadas apenas quanto ao volume do ejaculado. Os machos Saanen produziram maior

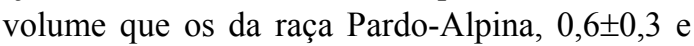
$0,5 \pm 0,3 \mathrm{ml}$, respectivamente. Estes resultados diferem daqueles descritos por Karagiannidis et al. (2000), que trabalharam com animais das mesmas raças, nascidos e criados na Grécia, ao observaram superioridade dos machos PardoAlpina em algumas características seminais, incluindo o volume do ejaculado, quando comparados com machos Saanen 1,27 $\pm 0,03$ e $1,15 \pm 0,03$, respectivamente. $O$ volume do ejaculado foi inferior aos dos machos Saanen e Pardo-Alpina criados no Reino Unido (Ahmad e Noakes, 1996) e equivalente aos dos machos cruzados Moxotó-Pardo-Alpina e Pardo-Alpina, criados no semi-árido nordestino (Santos e Simplício, 2000; Machado et al., 2000).

Embora o estresse calórico possa afetar negativamente várias características do ejaculado, o principal indício de diminuição da qualidade espermática tem sido a incidência de espermatozóides morfologicamente anormais, predominando os defeitos de gota citoplasmática proximal, de cauda enrolada e de cabeça decapitada (Kishore e Rao, 1983; Nunes et al., 1988; Santos e Simplício, 2000; Chemineau, 2004). Nesta pesquisa, não houve alteração do número de células anormais em função do estresse calórico (Tab. 3) nem predominância de 
algum defeito específico, resultados contrastantes com os da literatura citada. Isto ocorreu provavelmente devido às diferenças de procedimento adotadas para provocar o estresse calórico e à sua duração (Kishore e Rao, 1983; Epperson e Zalesky, 1995; Kraemer, 2000; Kastelic et al., 2000; Santos e Simplício, 2000; Moreira et al., 2001). Em pesquisas que utilizaram a técnica de insulação escrotal, o aparecimento de defeitos espermáticos, característicos de estresse calórico variou de uma a quatro semanas após a colocação da bolsa de insulação nas espécies bovina (Epperson e Zalesky, 1995; Kastelic et al., 2000), ovina (Moreira et al., 2001) e caprina (Kishore e Rao, 1983; Santos e Simplício, 2000). Ao contrário das pesquisas mencionadas, a metodologia deste experimento baseou-se na simulação do estresse calórico em câmara bioclimática por um período de 30 dias, tempo provavelmente insuficiente para o aparecimento dessas anormalidades. Além disso, o estresse calórico em câmara bioclimática nem sempre é capaz de reproduzir os efeitos deletérios da alta temperatura ambiente sobre as características seminais. Kraemer (2000), ao estudar o efeito da temperatura ambiente em touros mantidos em regiões subtropicais e simulados em câmara bioclimática, observou que o estresse calórico não foi suficiente para produzir efeitos negativos na qualidade espermática, como os observados nos animais mantidos em regime de pastejo.

Em regiões tropicais, a elevada temperatura ambiente parece ser o principal fator climático determinante das variações nas características quanti-qualitativas do sêmen caprino (Berbigier, 1988; Nunes et al., 1988). Neste trabalho, como o efeito do fotoperíodo foi neutralizado durante todo o período experimental e pelo fato de já existirem indícios de que em áreas subtropicais, como na região Sudeste do Brasil, os caprinos podem apresentar oscilações anuais na produção espermática em função do fotoperíodo (Espeschit, 1998), a temperatura ambiente parece ser a principal causa das variações observadas nas características seminais avaliadas.

\section{REFERÊNCIAS BIBLIOGRÁFICAS}

AGRICULTURAL and food research council. The nutrition of goat. Wallingford, $\mathrm{Cab}$ International, 1998. 118p.
AHMAD, N.; NOAKES, D.E. Seasonal variations in the semen quality of young British goats. Br. Vet. J., v.2, p.225-236, 1996.

ARRUDA, F.A.V.; PANT, K.P. Tolerância ao calor de caprinos e ovinos sem lã em Sobral. Pesq. Agropec. Bras., v.19, p.379-385, 1984.

BERBIGIER, P. Bioclimatologie des ruminants domestiques em zone tropicale. Ed. Paris: INRA, 1988. 237p.

BLOM, E. The ultra-structure of some characteristics sperm defects and a proposal for a new classification of bull spermogram. Norw. Vet. Med., v.25, p.383-391, 1973.

CHEMINEAU P. Medio ambiente y reproducción animal. 29/06/2004. <http://www. fao.org/ag/Aga/AGAP/WAR/warall/v1650b/v16 50b04.htm>.

CHEMINEAU P. Sexual behaviour and gonadal activity during the year in the tropical Creole meat goat. II. Male mating behaviour, testis diameter, ejaculate characteristics and fertility. Reprod. Nutr. Develop., v.26, p.453-460, 1986.

CORTEEL, J.M. Production de sperme chez le bouc: variation saisonnière de la quantite et de la qualite du sperme récolté selon l'âge des animaux. C.R. JOURNEES DE LA RECHERCHE OVINE ET CAPRINE, Paris: INRA - ITOVIC, 1975. v.1, p.4-17.

DELGADILLO, J.A.; LEBOEUF, B.; CHEMINEAU, P. Abolition of seasonal variations in semen quality and maintenance of sperm fertilizing ability by photoperiodic cycles in goat bucks. Small Rum. Res., v.9, p.47-59, 1992.

EPPERSON, B., ZALESKY, D. Effects of high heat and humidity on reproduction in cattle. Ex. Ex., 2018, 1995. http://agbiopubs.sdstate.edu/ articles/ExEx2018.pdf

ESPESCHIT, C.J.B. Alternativas para controle da estacionalidade reprodutiva de cabras leiteiras. In: ENCONTRO NACIONAL PARA O DESENVOLVIMENTO DA ESPÉCIE CAPRINA, 5., 1998, Botucatu. Anais..., 1998. p.7-33.

HIBBERT, L.M.; RODRIGUES, H.D.; NOBLE, R.C. et al. Effects of age and season on sperm abnormalities in Nubian goats. Anat. Histol. Embryol., v.15, p.173, 1986. 
KARAGIANNIDIS, A.; VARSAKELI, S.; KARATZAS, G. Characteristics and seasonal variations in the semen of Alpine Saanen and Damasco goat bucks born and raised in Greece. Small Rum. Res, v.37, p.125-130, 2000.

KASTELIC, J.P.; COOK, R.B.; COULTER, G.H. Scrotal/testicular thermoregulation in bulls. In: CHENOWETH, P.J. Topics in bull fertility. International Veterinary Information Service, 2000 (www.ivis.org).

KISHORE, P.N.; RAO, A.R. Effect of induced testicular degeneration on characteristics of bucks. Ind. Vet. J., v.60, p.281-286, 1983.

KRAEMER, T. The influence of high ambient temperature on different parameters of semen, biochemical and endocrine parameters in bulls in a climatic chamber and in subtropical climate. Berlin: FU Berlin, 2000. http://www.diss.fuberlin.de/2000/83/indexe.html

MACHADO, R.; FREITAS, A.R.; SIMPLÍCIO, A.A. et al. Flutuações sazonais e efeitos de raça no sêmen caprino. In: REUNIÃO ANUAL DA SOCIEDADE BRASILEIRA DE ZOOTECNIA, 27., Viçosa, 2000. Anais..., Viçosa: SBZ, 2000. CDROM.

MOREIRA, E.P.; MOURA, A.A.A.; ARAÚJO, A.A. Efeitos da insulação escrotal sobre a biometria testicular e parâmetros seminais em carneiros da raça Santa Inês criados no Estado do Ceará. Rev. Bras. Zootec., v.6, p.1704-1711, 2001.

NUNES, J.F. Fatores que influenciam os aspectos quanti-qualitativos do sêmen de caprinos no Nordeste do Brasil. Rev. Bras. Reprod. Anim., v.12, p.77-83, 1988.

PELLETIER, J.; CHEMINEAU, P.; DELGADILLO, J.A. Seasonality of sexual activity and its photoperiodic control in the adult ram and he-goat. In: INTERNATIONAL CONGRESS ON ANIMAL REPRODUCTION AND AI, 11., 1988, Dublin. Proceedings... Dublin, 1988. v.5, p.211-219.

PÉREZ, B.; MATEOS, E. Effect of photoperiod on semen production and quality in bucks of Verata and Malagueña breeds. Small Rum. Res., v.22, p.163-168, 1996.

ROCA, J.; MARTÍNEZ, E.; VÁSQUEZ, J.M.; COY, P. Characteristics and seasonal variations in the semen of Murciano-Granadina goats in the Mediterranean area. Anim. Reprod. Sci., v.29, p.255-262, 1992.

SANTOS, D.O.; SIMPLÍCIO, A.A. Parâmetros escroto-testiculares e de sêmen em caprinos adultos submetidos a insulação escrotal. Pesq. Agropec. Bras., v.35, p.1835-1841, 2000.

SAUMANDE, J.; ROUGER, Y. Variations saisonnieres des taux d'androgens dans le plasma de sang péripherique chez le bouc. CR. Acad. SC., v.274, p.89-92, 1984.

TURNER, H.G. Genetic variation of rectal temperature in cows and its relationship to fertility. Anim. Prod., v.35, p.401-412, 1982.

URIBE-VELÁSQUEZ, L.F.; OBA, E; BRASIL, L.H.A. Concentrações plasmáticas de cortisol, hormônios tiroídeanos, metabólitos lipídicos e temperatura de cabras Pardo-Alpinas submetidas ao estresse térmico. Rev. Bras. Zootec., v.27, p.1123-1130, 1998.

VALLE, A.; FUENTES, A.; PUERTA, M. Influencia de factores climáticos sobre loas características seminales de toros Holstein y Pardo Suizo nacidos en el trópico. Rev. Fac. Agron., v.22, p.52-61, 2005. 